\title{
Pion radiative weak decays in nonlocal chiral quark models
}

\author{
D. Gómez Dumm ${ }^{\mathrm{a}, \mathrm{b}, *}$, S. Noguera ${ }^{\mathrm{c}}$, N.N. Scoccola ${ }^{\mathrm{b}, \mathrm{d}, \mathrm{e}}$ \\ a IFLP, CONICET - Dpto. de Física, Universidad Nacional de La Plata, C.C. 67, 1900 La Plata, Argentina \\ b CONICET, Rivadavia 1917, 1033 Buenos Aires, Argentina \\ c Departamento de Física Teórica and Instituto de Física Corpuscular, Universidad de Valencia - CSIC, E-46100 Burjassot (Valencia), Spain \\ d Physics Department, Comisión Nacional de Energía Atómica, Av. Libertador 8250, 1429 Buenos Aires, Argentina \\ e Universidad Favaloro, Solís 453, 1078 Buenos Aires, Argentina
}

\section{A R T I C L E I N F O}

\section{Article history:}

Received 3 December 2010

Received in revised form 14 February 2011

Accepted 1 March 2011

Editor: J.-P. Blaizot

\section{Keywords:}

Nonlocal quark models

Pion radiative weak decays

\begin{abstract}
A B S T R A C T
We analyze the radiative pion decay $\pi^{+} \rightarrow e^{+} v_{e} \gamma$ within nonlocal chiral quark models that include wave function renormalization. In this framework we calculate the vector and axial-vector form factors $F_{V}$ and $F_{A}$ at $q^{2}=0$ - where $q^{2}$ is the $e^{+} v_{e}$ squared invariant mass - and the slope $a$ of $F_{V}\left(q^{2}\right)$ at $q^{2} \rightarrow 0$. The calculations are carried out considering different nonlocal form factors, in particular those taken from lattice QCD evaluations, showing a reasonable agreement with the corresponding experimental data. The comparison of our results with those obtained in the (local) NJL model and the relation of $F_{V}$ and $a$ with the form factor in $\pi^{0} \rightarrow \gamma^{*} \gamma$ decays are discussed.
\end{abstract}

(c) 2011 Elsevier B.V. All rights reserved.

The radiative pion decay $\pi^{+} \rightarrow e^{+} v_{e} \gamma$ is a very interesting process from different points of view. According to the standard description, the corresponding decay amplitude consists of the inner bremsstrahlung (IB) and structure-dependent (SD) terms. The IB contribution corresponds to the situation in which the photon is radiated by the electrically charged external legs, either pion or lepton, while the SD terms are associated with the photon emission from intermediate states generated by strong interactions. The latter can be parameterized through the introduction of vector and axial-vector form factors, $F_{V}\left(q^{2}\right)$ and $F_{A}\left(q^{2}\right)$, respectively, where $q^{2}$ is the squared invariant mass of the $e^{+} v_{e}$ pair [1]. Since $\pi^{+} \rightarrow e^{+} v_{e}$ is helicity suppressed, same happens to the IB terms that contribute to its radiative counterpart, and, consequently, $\pi^{+} \rightarrow e^{+} v_{e} \gamma$ turns out to be an appropriate channel to uncover the nonperturbative SD amplitude. Recent measurements [2] of the $\pi^{+} \rightarrow e^{+} \nu_{e} \gamma$ branching ratio over a wide region of phase space yields $F_{V}(0)=0.0258(17), F_{A}(0)=0.0117(17)$ and $a=0.10(6)$, where $a$ is related to the dependence of $F_{V}$ on $q^{2}$ parameterized as

$$
F_{V}\left(q^{2}\right) \simeq F_{V}(0)\left(1+a q^{2} / m_{\pi^{+}}^{2}\right) \text { for } q^{2} \ll m_{\pi^{+}}^{2} .
$$

From the point of view of the physics of weak interactions, this determination has provided a further check of conserved-vector-current (CVC) hypothesis. In fact, the value of $F_{V}(0)$ given above is in good agreement with that extracted from the analysis of the $\pi^{0} \rightarrow \gamma \gamma$ decay [3]. Moreover, as stressed in Ref. [2], a good description of the data appears to be possible without the need to include extra tensor contributions that arise in several extensions of the Standard Model suggested in the literature [4,5]. From the side of strong interaction physics, the pion radiative decays have been analyzed using Chiral Perturbation Theory [6] and effective meson Lagrangian methods [7]. However, there still remains the question of how the associated form factors are connected to the underlying quark structure. Due to the nonperturbative nature of the quark-gluon interactions in the low-energy domain, to address this issue one is forced to deal with models that treat quark interactions in some effective way. Among these, the Nambu-Jona-Lasinio (NJL) model has been widely used as a schematic effective theory for QCD [8-10], allowing e.g. the description of light mesons as fermion-antifermion composite states. In the NJL model quarks interact through a local, chiral invariant four-fermion coupling. The corresponding predictions for the vector and axial-vector form factors at $q^{2}=0$ have been calculated in Ref. [11], yielding $F_{V}(0)=0.0244$ and $F_{A}(0)=0.0241$. An extension of that calculation leads to $a=0.032$. As we see, while the predictions in the vector sector are in reasonable agreement with the measured values (the prediction for $a$ being slightly below the empirical range), the calculated value of $F_{A}(0)$ is a factor 2 too large. As a way to improve upon the NJL model, extensions which include nonlocal interactions have been proposed (see Ref. [12] and references therein). In fact,

\footnotetext{
* Corresponding author at: IFLP, CONICET - Dpto. de Física, Universidad Nacional de La Plata, C.C. 67, 1900 La Plata, Argentina.

E-mail address: dumm@fisica.unlp.edu.ar (D. Gómez Dumm).
} 
nonlocality arises naturally in several well-established approaches to low energy quark dynamics. This is e.g. the case of the instanton liquid model [13] and the Schwinger-Dyson resummation techniques [14], and also lattice QCD calculations [15-17] indicate that quark interactions should act over a certain range in momentum space. Indeed, nonlocal chiral quark models have been successfully applied to study different hadron observables [18-26]. The aim of the present work is to investigate the predictions of this type of models for the measured quantities associated to vector and axial-vector form factors in $\pi^{+} \rightarrow e^{+} \nu_{e} \gamma$ decays.

In general, the amplitude for the process $\pi^{+} \rightarrow e^{+} \nu_{e}(q)+\gamma(k)$ can be written as [1]

$$
\mathcal{M}=\frac{G_{F}}{\sqrt{2}} e \cos \theta_{C} \varepsilon_{\mu}\left[\sqrt{2} f_{\pi}\left\{(q+k)^{\alpha} L_{\alpha \mu}-l^{\nu}\left[g_{\mu \nu}+\frac{q_{\mu} q_{\nu}}{(q \cdot k)}\right]\right\}+l^{\nu}\left\{-i \epsilon_{\mu \nu \alpha \beta} k^{\alpha} q^{\beta} \frac{F_{V}\left(q^{2}\right)}{m_{\pi}}+\left[q_{\mu} k_{\nu}-g_{\mu \nu}(q \cdot k)\right] \frac{F_{A}\left(q^{2}\right)}{m_{\pi}}\right\}\right],
$$

with $(q+k)^{2}=m_{\pi}^{2}, k^{2}=0$. Here $G_{F}$ and $\theta_{C}$ stand for the Fermi constant and the Cabibbo angle, respectively; $\varepsilon_{\mu}$ is the photon polarization vector, $l_{\mu}$ is the lepton current, $L_{\alpha \mu}$ is a lepton tensor, and $F_{V}\left(q^{2}\right)$ and $F_{A}\left(q^{2}\right)$ denote the vector and axial-vector hadronic form factors mentioned above. In this work we are interested in the study of the predictions for the measured quantities associated with these form factors in the context of nonlocal chiral models. We consider SU(2) chiral models that include wave function renormalization, defined by the following Euclidean action [24,26]

$$
S_{E}=\int d^{4} x\left\{\bar{\psi}(x)\left(-i \not \partial+m_{c}\right) \psi(x)-\frac{G_{S}}{2}\left[j_{a}(x) j_{a}(x)+j_{P}(x) j_{P}(x)\right]\right\} .
$$

Here $m_{c}$ is the current quark mass, which is assumed to be equal for $u$ and $d$ quarks, while the nonlocal currents $j_{a}(x), j_{P}(x)$ are given by

$$
j_{a}(x)=\int d^{4} z g(z) \bar{\psi}\left(x+\frac{z}{2}\right) \Gamma_{a} \psi\left(x-\frac{z}{2}\right), \quad j_{P}(x)=\int d^{4} z f(z) \bar{\psi}\left(x+\frac{z}{2}\right) \frac{i \overleftrightarrow{\not}}{2 \varkappa_{p}} \psi\left(x-\frac{z}{2}\right),
$$

where $\Gamma_{a}=\left(\mathbb{1}, i \gamma_{5} \vec{\tau}\right)$ and $u\left(x^{\prime}\right) \overleftrightarrow{\partial} v(x)=u\left(x^{\prime}\right) \partial_{x} v(x)-\partial_{x^{\prime}} u\left(x^{\prime}\right) v(x)$. The functions $g(z)$ and $f(z)$ in Eq. (4) are nonlocal covariant form factors characterizing the corresponding interactions. In what follows it is convenient to Fourier transform $g(z)$ and $f(z)$ into momentum space. Note that Lorentz invariance implies that the Fourier transforms $g_{p}$ and $f_{p}$ can only be functions of $p^{2}$.

In order to deal with meson degrees of freedom, one can perform a standard bosonization of the theory. This is done by considering the corresponding partition function $\mathcal{Z}=\int \mathcal{D} \bar{\psi} \mathcal{D} \psi \exp \left[-S_{E}\right]$, and introducing auxiliary fields $\sigma_{1}(x), \sigma_{2}(x), \vec{\pi}(x)$, where $\sigma_{1,2}(x)$ and $\vec{\pi}(x)$ are scalar and pseudoscalar mesons, respectively. An effective bosonized action is obtained once the fermion fields are integrated out. To treat that bosonic action we assume, as customary, that $\sigma_{1,2}$ fields have nontrivial translational invariant mean field values $\bar{\sigma}_{1,2}$, while the mean field values of pseudoscalar fields $\pi_{i}$ are zero. Thus we write

$$
\sigma_{1}(x)=\bar{\sigma}_{1}+\delta \sigma_{1}(x), \quad \sigma_{2}(x)=\varkappa_{p} \bar{\sigma}_{2}+\delta \sigma_{2}(x), \quad \vec{\pi}(x)=\delta \vec{\pi}(x) .
$$

Replacing in the bosonized effective action and expanding in powers of meson fluctuations we get

$$
S_{E}^{\text {bos }}=S_{E}^{\mathrm{MFA}}+S_{E}^{\text {quad }}+\cdots
$$

Here the mean field action per unit volume reads

$$
\frac{S_{E}^{\mathrm{MFA}}}{V^{(4)}}=\frac{1}{2 G_{S}}\left(\bar{\sigma}_{1}^{2}+\varkappa_{p}^{2} \bar{\sigma}_{2}^{2}\right)-4 N_{c} \int \frac{d^{4} p}{(2 \pi)^{4}} \ln \left[\frac{z_{p}}{-\not p+m_{p}}\right]^{-1},
$$

with

$$
z_{p}=\left(1-\bar{\sigma}_{2} f_{p}\right)^{-1}, \quad m_{p}=z_{p}\left(m_{c}+\bar{\sigma}_{1} g_{p}\right) .
$$

The minimization of $S_{E}^{\mathrm{MFA}}$ with respect to $\bar{\sigma}_{1,2}$ leads to the corresponding gap equations. The quadratic terms can be written as

$$
S_{E}^{\text {quad }}=\frac{1}{2} \int \frac{d^{4} p}{(2 \pi)^{4}} \sum_{M=\sigma, \sigma^{\prime}, \pi} G_{M}\left(p^{2}\right) \delta M(p) \delta M(-p),
$$

where $\sigma$ and $\sigma^{\prime}$ fields are meson mass eigenstates, defined in such a way that there is no $\sigma-\sigma^{\prime}$ mixing at the level of the quadratic action. The explicit expressions of $G_{M}\left(p^{2}\right)$, as well as those of the gap equations mentioned above, can be found in Ref. [26]. Meson masses can be obtained by solving the equation $G_{M}\left(-m_{M}^{2}\right)=0$, while on-shell meson-quark coupling constants $g_{M q \bar{q}}$ are given by

$$
g_{M q \bar{q}}-2=\left.\frac{d G_{M}\left(p^{2}\right)}{d p^{2}}\right|_{p^{2}=-m_{M}^{2}} .
$$

As in Ref. [26], we will consider here different functional dependencies for the form factors $g_{p}$ and $f_{p}$. First, we consider a relatively simple case in which there is no wave function renormalization of the quark propagator, i.e. $f_{p}=0, z_{p}=1$, and we take an often used exponential parameterization for $g_{p}$,

$$
g_{p}=\exp \left(-p^{2} / \Lambda_{0}^{2}\right)
$$


The model parameters $m_{c}, G_{S}$ and $\Lambda_{0}$ are determined by fitting the pion mass and decay constant to their empirical values $m_{\pi}=139$ MeV and $f_{\pi}=92.4 \mathrm{MeV}$, and fixing the chiral condensate to the phenomenologically acceptable value $\langle\bar{q} q\rangle^{1 / 3}=-240 \mathrm{MeV}$. In what follows we refer to this choice of model parameters as Set A. Second, we consider a more general case that includes the wave function renormalization of the quark propagator. We keep the exponential shape (10) for the form factor $g_{p}$ and assume also an exponential form for $f_{p}$, namely

$$
f_{p}=\exp \left(-p^{2} / \Lambda_{1}^{2}\right)
$$

Note that the range (in momentum space) of the nonlocality in each channel is determined by the parameters $\Lambda_{0}$ and $\Lambda_{1}$, respectively. As in the previous case, model parameters are determined so as to reproduce the desired values of $m_{\pi}, f_{\pi}$ and $\langle\bar{q} q\rangle^{1 / 3}$. The form factor $f_{p}$ introduces now an additional free parameter $\Lambda_{1}$, consequently we introduce as a fourth requirement the condition $z_{p}(0)=0.7$, which is within the range of values suggested by recent lattice calculations [15,17]. This choice of model parameters and form factors will be referred to as parameterization Set B. Finally, we consider a different functional form for the form factors, given by

$$
g_{p}=\frac{1+\alpha_{z}}{1+\alpha_{z} f_{z}(p)} \frac{\alpha_{m} f_{m}(p)-m \alpha_{z} f_{z}(p)}{\alpha_{m}-m \alpha_{z}}, \quad f_{p}=\frac{1+\alpha_{z}}{1+\alpha_{z} f_{z}(p)} f_{z}(p)
$$

where

$$
f_{m}(p)=\left[1+\left(p^{2} / \Lambda_{0}^{2}\right)^{3 / 2}\right]^{-1}, \quad f_{z}(p)=\left[1+\left(p^{2} / \Lambda_{1}^{2}\right)\right]^{-5 / 2}
$$

As shown in Ref. [26], taking $m_{c}=2.37 \mathrm{MeV}, \alpha_{m}=309 \mathrm{MeV}, \alpha_{z}=-0.3, \Lambda_{0}=850 \mathrm{MeV}$ and $\Lambda_{1}=1400 \mathrm{MeV}$ one can very well reproduce the momentum dependence of mass and renormalization functions obtained in lattice calculations, as well as the physical values of $m_{\pi}$ and $f_{\pi}$. In what follows we will refer to this choice of model parameters as parameterization Set $\mathrm{C}$. The parameter values for all three parameter sets, as well as the corresponding predictions for several meson properties, can be found in Ref. [26].

In order to derive the form factors we are interested in, one should "gauge" the effective action $S_{E}$ by introducing the electromagnetic field $A_{\mu}(x)$ and the charged weak fields $W_{\mu}^{ \pm}(x)$. For a local theory this "gauging" procedure is usually done by performing the replacement

$$
\partial_{\mu} \rightarrow \partial_{\mu}+i G_{\mu}(x)
$$

where

$$
G_{\mu}(x)=\frac{e}{2}\left(\frac{1}{3}+\tau^{3}\right) A_{\mu}(x)+g_{W} \frac{1-\gamma_{5}}{2} \frac{\tau^{+} W_{\mu}^{+}(x)+\tau^{-} W_{\mu}^{-}(x)}{\sqrt{2}},
$$

with $g_{W}^{2} /\left(8 M_{W}^{2}\right)=G_{F} \cos \theta_{C} / \sqrt{2}$ and $\tau^{ \pm}=\left(\tau^{1} \pm i \tau^{2}\right) / 2$. In the present case - owing to the nonlocality of the involved fields - one has to perform additional replacements in the interaction terms, namely

$$
\psi(x-z / 2) \rightarrow W_{G}(x, x-z / 2) \psi(x-z / 2), \quad \psi^{\dagger}(x+z / 2) \rightarrow \psi^{\dagger}(x+z / 2) W_{G}(x+z / 2, x) .
$$

Here $x$ and $z$ are the variables appearing in the definitions of the nonlocal currents [see Eq. (4)], and the function $W_{G}(x, y)$ is defined by

$$
W_{G}(x, y)=P \exp \left[i \int_{x}^{y} d r_{\mu} G_{\mu}(r)\right]
$$

where $r$ runs over an arbitrary path connecting $x$ with $y$.

Once the gauged effective action is built, the explicit expressions for the vector and axial-vector form factors can be obtained by expanding to leading order in the product $\delta \pi^{+} A_{\mu} W_{v}^{+}$. This is a rather lengthy calculation that can be simplified by considering $q^{2} \ll m_{\pi^{+}}^{2}$, as needed to make predictions for the measured quantities $F_{V}(0), F_{A}(0)$ and $a$.

The vector form factor is obtained from the triangle diagram represented in Fig. 1(a). As stated, $F_{V}\left(q^{2}\right)$ can be expanded at leading order in $q^{2}$ as in Eq. (1), just changing $q^{2} \rightarrow-q^{2}$ since we are working in Euclidean space. Performing such an expansion we obtain

$$
\frac{F_{V}(0)}{m_{\pi^{+}}}=\frac{\sqrt{2} g_{\pi q \bar{q}} N_{c}}{3} \int \frac{d^{4} \ell}{(2 \pi)^{4}} g_{\ell_{0}} \frac{\left(z_{\ell}+z_{\ell_{K}}\right)\left(z_{\ell}+z_{\ell_{Q}}\right)}{D_{\ell} D_{\ell_{K}} D_{\ell_{Q}} z_{\ell}}\left[m_{\ell}-\frac{\ell^{2}}{2}\left(\frac{m_{\ell_{Q}}-m_{\ell}}{Q \cdot \ell}-\frac{m_{\ell_{K}}-m_{\ell}}{K \cdot \ell}\right)\right],
$$

where $K$ and $Q$ are the photon and $e^{+} v_{e}$ pair momenta, respectively, taken at $q^{2}=0$. In Eq. (18) we have used the definitions $D_{\ell}=$ $\ell^{2}+m_{\ell}^{2}, \ell_{Q}=\ell+Q, \ell_{K}=\ell-K$ and $\ell_{0}=\left(\ell_{Q}+\ell_{K}\right) / 2$. For convenience we also define $\ell_{Q}^{ \pm}=\ell \pm Q / 2, \ell_{K}^{ \pm}=\ell \pm K / 2$ and $\ell_{K}^{ \pm}=$ $\ell \pm(K+Q) / 2$, which will be used below.

The calculation of $a$ is somewhat more involved. In particular, the result depends on the integration path appearing in the nonlocal contribution to the quark-gauge boson vertices [see Eq. (17)]. One obtains:

$$
a=-\frac{m_{\pi^{+}}}{F_{V}(0)} \sqrt{2} \frac{N_{c}}{3} g_{\pi q \bar{q}} \int \frac{d^{4} \ell}{(2 \pi)^{4}} g_{\ell_{0}} \frac{\left(z_{\ell}+z_{\ell_{K}}\right)\left(z_{\ell}+z_{\ell_{Q}}\right)}{D_{\ell} D_{\ell_{K}} D_{\ell_{Q}} z_{\ell}} \mathcal{B}(\ell, K, Q)
$$

where 


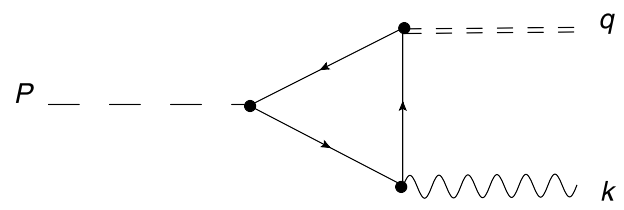

(a)

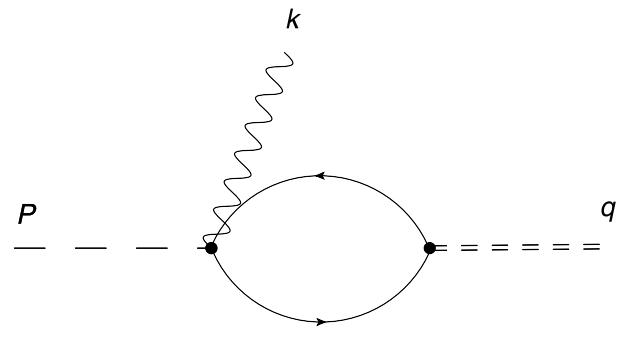

(b)

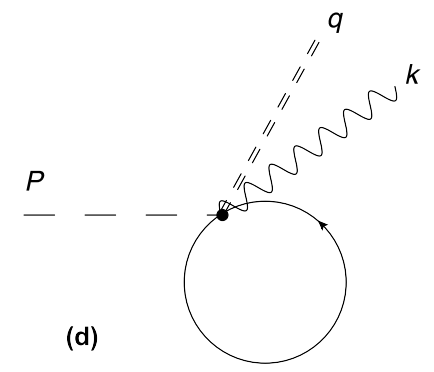

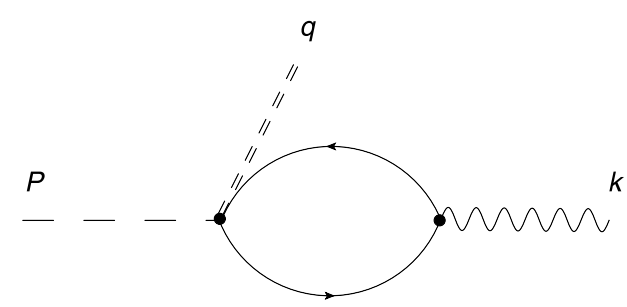

(c)

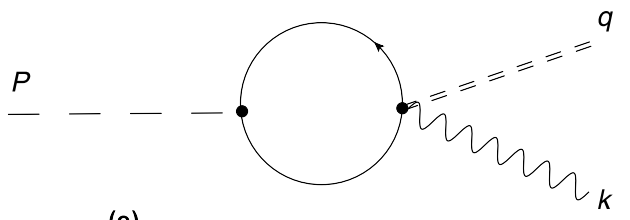

(e)

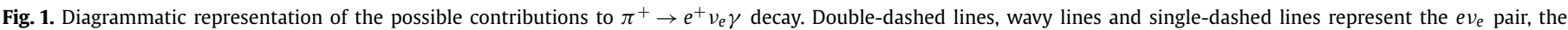

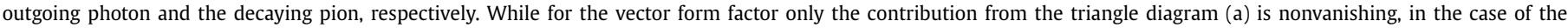
axial-vector form factor all five diagrams contribute.

$$
\begin{aligned}
\mathcal{B}(\ell, K, Q)= & {\left[m_{\ell}-\frac{\ell^{2}}{2}\left(\frac{m_{\ell_{Q}}-m_{\ell}}{Q \cdot \ell}-\frac{m_{\ell_{K}}-m_{\ell}}{K \cdot \ell}\right)\right] } \\
& \times 2\left[K \cdot \ell_{Q}\left(\frac{D_{\ell_{Q}}^{\prime}}{D_{\ell_{Q}}}-\frac{z_{\ell_{Q}}^{\prime}}{z_{\ell}+z_{\ell_{Q}}}\right)+K \cdot \ell_{K}\left(\frac{D_{\ell_{K}}^{\prime}}{D_{\ell_{K}}}-\frac{z_{\ell_{K}}^{\prime}}{z_{\ell}+z_{\ell_{K}}}\right)-K \cdot \ell_{0} \frac{g_{\ell_{0}}^{\prime}}{g_{\ell_{0}}}\right] \\
& +\ell^{2}\left(\frac{K \cdot \ell_{Q}}{Q \cdot \ell} m_{\ell_{Q}}^{\prime}-m_{\ell_{K}}^{\prime}\right)+\frac{1}{2}\left(\frac{2 K \cdot \ell}{K \cdot Q}-\frac{\ell^{2}}{K \cdot \ell}\right)\left(m_{\ell_{K}}-m_{\ell}\right) \\
& +\left(K \cdot \ell-\frac{\ell^{2}}{2} \frac{K \cdot Q}{Q \cdot \ell}\right)\left[\frac{K \cdot \ell_{Q}}{Q \cdot \ell K \cdot Q}\left(m_{\ell_{Q}}-m_{\ell}\right)+\frac{2 z_{\ell_{Q}} z_{\ell}}{z_{\ell_{Q}}+z_{\ell}}\left(\alpha_{\ell, Q}+\frac{m_{\ell_{Q}}+m_{\ell}}{2} \beta_{\ell, Q}\right)\right] .
\end{aligned}
$$

Here primes stand for derivatives, e.g. $g_{\ell}^{\prime}=d g_{\ell} / d \ell^{2}$. The functions $\alpha_{\ell, Q}$ and $\beta_{\ell, Q}$ are, in general, path dependent. Here, for simplicity, we choose to use the "straight line path" for which they read

$$
\alpha_{\ell, Q}=\bar{\sigma}_{1} \int_{-1}^{1} d \lambda \lambda g_{\ell_{Q}^{+}+\frac{\lambda}{2} Q}^{\prime}, \quad \beta_{\ell, Q}=\bar{\sigma}_{2} \int_{-1}^{1} d \lambda \lambda f_{\ell_{Q}^{+}+\frac{\lambda}{2} Q}^{\prime}
$$

The axial-vector form factor receives not only a contribution from the triangle diagram in Fig. 1(a) (as occurs in the local NJL model) but also from other diagrams, which are represented in Figs. 1(b)-(e). Thus, for $q^{2}=0$ one has

$$
F_{A}(0)=\left.\sum_{\alpha=a}^{e} F_{A}(0)\right|_{\alpha}
$$

The contribution from the triangle diagram is given by

$$
\left.\frac{F_{A}(0)}{m_{\pi^{+}}}\right|_{a}=-\frac{\sqrt{2} g_{\pi q q} N_{c}}{(K \cdot Q)^{2}} \int \frac{d^{4} \ell}{(2 \pi)^{4}} g_{\ell_{0}} \frac{z_{\ell_{Q}} z_{\ell_{K}}}{D_{\ell} D_{\ell_{Q}} D_{\ell_{K}}} \mathcal{A}_{a},
$$

where 


$$
\begin{aligned}
\mathcal{A}_{a}= & 2\left(4-\frac{K \cdot Q}{K \cdot \ell Q \cdot \ell} \ell^{2}\right)\left\{\left(\ell_{Q} \cdot \ell_{K}+m_{\ell_{Q}} m_{\ell_{K}}\right)\left[\left(\frac{m_{\ell_{Q}^{+}}}{z_{\ell_{Q}^{+}}}+\frac{m_{\ell_{K}^{-}}}{z_{\ell_{K}^{-}}}-\frac{m_{\ell}}{z_{\ell}}\right) D_{\ell}+K \cdot Q \frac{m_{\ell}\left(z_{\ell_{Q}}+z_{\ell}\right)\left(z_{\ell_{K}}+z_{\ell}\right)}{4 z_{\ell} z_{\ell_{Q}} z_{\ell_{K}}}\right]\right. \\
& \left.-\frac{z_{\ell} m_{\ell_{K}^{-}}}{z_{\ell_{Q}} z_{\ell_{K}^{-}}}\left(\ell \cdot \ell_{K}+m_{\ell} m_{\ell_{K}}\right) D_{\ell_{Q}}-\frac{z_{\ell} m_{\ell_{Q}^{+}}}{z_{\ell_{K}} z_{\ell_{Q}^{+}}}\left(\ell \cdot \ell_{Q}+m_{\ell} m_{\ell_{Q}}\right) D_{\ell_{K}}+\frac{z_{\ell} m_{\ell}}{z_{\ell_{K}} z_{\ell_{Q}}} D_{\ell_{Q}} D_{\ell_{K}}\right\} \\
& +K \cdot Q\left(2-\frac{K \cdot Q}{K \cdot \ell Q \cdot \ell} \ell^{2}\right)\left(1+\frac{z_{\ell}}{z_{\ell_{Q}}}\right)\left(1+\frac{z_{\ell}}{z_{\ell_{K}}}\right) \\
& \times\left\{\frac{m_{\ell_{Q}^{+}}}{z_{\ell_{Q}^{+}}} \frac{\ell \cdot \ell_{K}+m_{\ell} m_{\ell_{K}}}{1+z_{\ell} / z_{\ell_{Q}}}+\frac{m_{\ell_{K}^{-}}}{z_{\ell_{K}^{-}}} \frac{\ell \cdot \ell_{Q}+m_{\ell} m_{\ell_{Q}}}{1+z_{\ell} / z_{\ell_{K}}}-\frac{m_{\ell}}{z_{\ell}}\left(\ell_{0}^{2}+m_{\ell_{Q}} m_{\ell_{K}}\right)\right\},
\end{aligned}
$$

while the remaining contributions are given by

$$
\left.\sum_{\alpha=b}^{e} \frac{F_{A}(0)}{m_{\pi^{+}}}\right|_{\alpha}=\frac{4 \sqrt{2} g_{\pi q \bar{q}} N_{c}}{(K \cdot Q)^{2}} \int \frac{d^{4} \ell}{(2 \pi)^{4}}\left[\left(\frac{K \cdot Q}{K \cdot \ell Q \cdot \ell} \frac{\ell^{2}}{2}-2\right)\left(\mathcal{A}_{b}+\mathcal{A}_{c}+\mathcal{A}_{d}\right)+\mathcal{A}_{e}\right],
$$

where

$$
\begin{aligned}
& \mathcal{A}_{b}=\left(g_{\ell}-g_{\ell_{K}^{-}}\right)\left[\frac{z_{\ell_{Q}^{+}} m_{\ell_{Q}^{+}}}{D_{\ell_{Q}^{+}}}+\frac{z_{\ell_{Q}^{-}} m_{\ell_{Q}^{-}}}{D_{\ell_{Q}^{-}}}-2 \frac{m_{\ell}}{z_{\ell}} \frac{z_{\ell_{Q}^{+}} z_{\ell_{Q}^{-}}}{D_{\ell_{Q}^{+}} D_{\ell_{Q}^{-}}}\left(\ell^{2}+m_{\ell_{Q}^{-}} m_{\ell_{Q}^{+}}\right)\right], \\
& \mathcal{A}_{c}=g_{\ell_{K}^{-}}\left[\frac{z_{\ell_{Q}^{+}} m_{\ell_{Q}^{+}}}{D_{\ell_{Q}^{+}}}-\frac{z_{\ell_{Q}^{-}} m_{\ell_{Q}^{-}}}{D_{\ell_{Q}^{-}}}\right], \\
& \mathcal{A}_{d}=\left(g_{\ell}-g_{\ell_{K}^{-}}\left[\frac{z_{\ell} m_{\ell}}{D_{\ell}}-\frac{z_{\ell_{Q}^{-}} m_{\ell_{Q}^{-}}}{D_{\ell_{Q}^{-}}}\right],\right. \\
& \mathcal{A}_{e}=\bar{\sigma}_{1} g_{\ell} \frac{z_{\ell_{K Q}^{+}} z_{\ell_{K Q}^{-}}}{D_{\ell_{K Q}^{+}} D_{\ell_{K Q}^{-}}}\left(\ell_{K Q}^{+} \cdot \ell_{K Q}^{-}+m_{\ell_{K Q}^{+}} m_{\ell_{K Q}^{-}}\right)\left[g_{\ell_{Q}^{+}}+g_{\ell_{K}^{-}}-g_{\ell_{0}}-g_{\ell}-\gamma(\ell, K, Q)\right]
\end{aligned}
$$

with

$$
\gamma(\ell, K, Q)=\frac{K \cdot Q}{2} \int_{0}^{1} d \lambda \frac{\left(\ell^{2}-2 \frac{K \cdot \ell Q \cdot \ell}{K \cdot Q}\right)\left(g_{\ell+\frac{\lambda}{2} Q}^{\prime}-g_{\ell_{K}^{-}+\frac{\lambda}{2} Q}^{\prime}\right)+g_{\ell+\frac{\lambda}{2} Q}-g_{\ell_{K}^{-}+\frac{\lambda}{2} Q}}{K \cdot\left(\ell+\frac{\lambda}{2} Q\right)}
$$

Note that, contrary to what happens with $F_{V}(0)$, the axial-vector form factor depends on the path even at $q^{2}=0$. This is due to the contribution of the diagram of Fig. 1(e). The expression for $\gamma(\ell, K, Q)$ given above corresponds to the "straight line path" choice.

Before discussing our predictions for the form factors associated with the charged pion radiative weak decay, let us note that one can also consider the related decay processes $\pi^{0} \rightarrow \gamma \gamma$ and $\pi^{0} \rightarrow e^{+} e^{-} \gamma$. The amplitude of these processes contains the $\pi^{0} \gamma \gamma$ vertex form factor $F^{\pi \gamma \gamma^{*}}\left(q^{2}\right)$, where $q^{2}$ is now the invariant mass of the virtual photon. As in the case of $F_{V}\left(q^{2}\right)$ in the decay $\pi^{+} \rightarrow e^{+} \nu \gamma$, one can perform an expansion for low $q^{2}$ :

$$
F^{\pi \gamma \gamma^{*}} \simeq F^{\pi \gamma \gamma^{*}}(0)\left(1+a^{\prime} q^{2} / m_{\pi^{0}}^{2}\right), \text { with } q^{2} \ll m_{\pi^{0}}^{2} .
$$

Experimental measurements lead to $F^{\pi \gamma \gamma^{*}}(0)=0.284(8) \mathrm{GeV}^{-1}$ and $a^{\prime}=0.032$ (4) [3]. Here it is important to recall that, in the chiral limit, the anomaly leads to the constraint $F^{\pi \gamma \gamma^{*}}(0)=1 /\left(4 \pi^{2} f_{\pi}\right) \simeq 0.274 \mathrm{GeV}^{-1}$. Moreover, using CVC and isospin arguments it is easy to prove that $F_{V}(0)=m_{\pi} F^{\pi \gamma \gamma^{*}}(0) / \sqrt{2}$. We stress that our model satisfies this relation as well as the anomaly constraint. In addition, it is easy to see that it leads to an analytical expression for $a^{\prime}$ which coincides with that given in Eq. (19) for $a$.

We discuss now our numerical results. In Table 1 we list the predicted values of $F_{V}(0)$ and the slope $a$ for the three parameterizations considered in this work. We also include the available empirical data, as well as the (local) NJL model predictions. In the case of $F_{V}(0)$, we observe that the predictions of all three parameterizations of the nonlocal model are in good agreement with the empirical value and with the value obtained in the NJL model. This is hardly surprising, given the chiral limit constraints mentioned above. Regarding the slope parameter we find a too small value for all three parameterizations. There is a small improvement by going from Set A to Set B (which implies the introduction of the wave function renormalization of the quark propagator), and a larger improvement is obtained by going from Set B to Set C. One of the peculiarities of the nonlocal model is that the results can be dependent on the path used in Eq. (17). As usual, we have chosen a straight line path for the calculations, obtaining the expressions given in Eq. (21) for the path dependent quantities $\alpha_{\ell, Q}$ and $\beta_{\ell, Q}$. To gauge the importance of this path dependence we have evaluated the contribution of the corresponding terms, obtaining that it represents less than $3 \%$ for parameterizations A and B and less than $0.5 \%$ for Set C. As for the predictions for $a^{\prime}$ we note that, even though in our model its analytical expression coincides with that for $a$, the corresponding numerical values are somewhat different since in the case of $a^{\prime}$ we evaluate Eq. (19) at $P^{2}=m_{\pi^{0}}^{2}$, whereas for $a$ it is evaluated at $P^{2}=m_{\pi^{+}}^{2}$. This dependence on the mass of the pion reduces the value of $a^{\prime}$ in comparison with that of $a$ by about $10 \%$ for Sets A and B and $5 \%$ for Set C. We observe in Table 1 that $a^{\prime}$ is better reproduced than $a$, but the large errors in the experimental determination of the latter prevent us to take definite conclusions. In fact, the discrepancies between our predictions for $a$ and $a^{\prime}$ and their experimental values are not unexpected, since no vector interaction has been included in our model. For example, using the results of Ref. [28] we obtain that the vector contribution to 
Table 1

Results for $F_{V}(0)$ and $a$. All results should be multiplied by $10^{-2}$. In column 5 we give the empirical values of $m_{\pi}+F^{\pi \gamma \gamma^{*}}(0) / \sqrt{2}$ and $a^{\prime}$. Note that in our model $a=a^{\prime}$. In column 6 we give the prediction of the local Nambu-Jona-Lasinio (NJL) model.

\begin{tabular}{lllllll}
\hline & Set A & Set B & Set C & Exp [2] & Exp $\left(\pi^{0} \rightarrow \gamma \gamma^{*}\right)[3]$ \\
\hline$F_{V}(0)$ & 2.697 & 2.693 & 2.695 & $2.58 \pm 0.17$ & $2.80 \pm 0.08$ & NJL \\
$a$ & 1.651 & 1.726 & 2.011 & $10 \pm 6$ & $3.2 \pm 0.4$ & 3.244 \\
\hline
\end{tabular}

Table 2

Results for $F_{A}(0)$. All results should be multiplied by $10^{-2}$. In column 5 we give the prediction of the local Nambu-Jona-Lasinio (NJL) model.

\begin{tabular}{lcccc}
\hline & Set A & Set B & Set C & Exp [2] \\
\hline$\left.F_{A}(0)\right|_{a}$ & 1.300 & 1.591 & 1.804 & 2.409 \\
$\left.F_{A}(0)\right|_{b}$ & 0.067 & 0.047 & 0.031 & - \\
$\left.F_{A}(0)\right|_{c}$ & -0.0002 & -0.0002 & -0.0001 & - \\
$\left.F_{A}(0)\right|_{d}$ & -0.044 & -0.036 & -0.026 & - \\
$\left.F_{A}(0)\right|_{e}$ & -0.003 & 0.013 & 0.017 & - \\
$F_{A}(0)$ & 1.319 & 1.614 & 1.825 & 2.409 \\
\hline
\end{tabular}

the $\pi^{0} \rightarrow \gamma \gamma^{*}$ process has the right order of magnitude and sign needed to account for these discrepancies. Here it is interesting to point out that the contribution from the vector channel could be different for $a$ and $a^{\prime}$, even preserving the isospin symmetry. This would be achieved if one has different interactions in the vector-isoscalar channel and in the vector-isovector channel. On the other hand, it can be seen that the NJL model is able to provide a very good prediction for $a^{\prime}$. However, it is worth to notice that in the NJL framework this value is essentially given by the relation $a^{\prime} \simeq m_{\pi}^{2} /\left(12 m_{q}^{2}\right)$ [27], and the value of $m_{q}$ (dressed quark mass) turns out to be quite dependent on model parameterizations. The NJL values quoted here correspond to a Pauli-Villars regularization.

In Table 2 we give our numerical results for $F_{A}(0)$, quoting the contribution of each diagram. We have also quoted in Table 2 the result obtained in the NJL model, where the triangle diagram is the only one that survives. We observe that although several diagrams contribute in the case of the nonlocal models, the triangle diagram turns out to be the dominant one. The contribution coming from diagrams (b) to (e) is less than $2 \%$ of the total result, in all three parameterizations. In particular, diagram (e), the only one with a path dependent term, contributes with less than $1 \%$ of the full result. It is interesting to mention that $F_{A}(0)$ has also been evaluated in the spectral quark model, obtaining $F_{A}(0) \sim F_{V}(0)$ as in the NJL model [29]. In this sense, the fact that nonlocal models of the type considered here lead to values of $F_{A}(0)$ which are significantly different from those of $F_{V}(0)$ appears as a quite important result. A similar conclusion has been obtained in a simplified calculation in the chiral limit [30]. Given the triangle diagram dominance mentioned above, the origin of this difference can be traced back to the different dressing of the $\gamma_{\mu}$ and $\gamma_{\mu} \gamma_{5}$ terms in the coupling of the $W$ to the quarks [26]. Comparing our prediction for $F_{A}(0)$ with the empirical value, we observe that the introduction of the nonlocality gives half of the difference needed in the case of Set $\mathrm{C}$ and is near to exhaust this difference for Set A, which gives the best result. In any case, as in the case of the slope of the vector form factor, additional effects that have been neglected in the present calculation might help in improving the agreement with the empirical value.

\section{Acknowledgements}

We would like to acknowledge useful discussions with J. Portolés. This work has been partially funded by the Spanish MCyT (and EU FEDER) under contract FPA2007-65748-C02-01, FPA2008-04810-E, FPA2010-21750-C02-01 and AIC10-D-000588, by Consolider Ingenio 2010 CPAN (CSD2007-00042), by Generalitat Valenciana: Prometeo/2009/129, by the European Integrated Infrastructure Initiative HadronPhysics2 (Grant No. 227431), by CONICET (Argentina) under grants \# PIP 00682 and PIP 02495, and by ANPCyT (Argentina) under grant \# PICT07 03-00818.

\section{References}

[1] M. Moreno, Phys. Rev. D 16 (1977) 720; D.A. Bryman, P. Depommier, C. Leroy, Phys. Rep. 88 (1982) 151.

[2] M. Bychkov, et al., Phys. Rev. Lett. 103 (2009) 051802, arXiv:0804.1815 [hep-ex].

[3] K. Nakamura, et al., Particle Data Group, J. Phys. G 37 (2010) 075021.

[4] M.V. Chizhov, Mod. Phys. Lett. A 8 (1993) 2753, arXiv:hep-ph/0401217; M.V. Chizhov, Phys. Part. Nucl. Lett. 2 (2005) 193, Pis'ma Fiz. Elem. Chast. Atom. Yadra 2 (4) (2005) 7, arXiv:hep-ph/0402105.

[5] A.A. Poblaguev, Phys. Rev. D 68 (2003) 054020, arXiv:hep-ph/0307166.

[6] B.R. Holstein, Phys. Rev. D 33 (1986) 3316;

J. Bijnens, P. Talavera, Nucl. Phys. B 489 (1997) 387, arXiv:hep-ph/9610269;

C.Q. Geng, I.L. Ho, T.H. Wu, Nucl. Phys. B 684 (2004) 281, arXiv:hep-ph/0306165.

[7] V. Mateu, J. Portoles, Eur. Phys. J. C 52 (2007) 325, arXiv:0706.1039 [hep-ph].

[8] U. Vogl, W. Weise, Prog. Part. Nucl. Phys. 27 (1991) 195.

[9] S.P. Klevansky, Rev. Mod. Phys. 64 (1992) 649.

[10] T. Hatsuda, T. Kunihiro, Phys. Rep. 247 (1994) 221.

[11] A. Courtoy, S. Noguera, Phys. Rev. D 76 (2007) 094026, arXiv:0707.3366 [hep-ph].

[12] G. Ripka, Quarks Bound by Chiral Fields, Oxford University Press, Oxford, 1997.

[13] T. Schafer, E.V. Shuryak, Rev. Mod. Phys. 70 (1998) 323, arXiv:hep-ph/9610451.

[14] C.D. Roberts, A.G. Williams, Prog. Part. Nucl. Phys. 33 (1994) 477, arXiv:hep-ph/9403224; C.D. Roberts, S.M. Schmidt, Prog. Part. Nucl. Phys. 45 (2000) S1, arXiv:nucl-th/0005064

[15] M.B. Parappilly, P.O. Bowman, U.M. Heller, D.B. Leinweber, A.G. Williams, J.B. Zhang, Phys. Rev. D 73 (2006) 054504, arXiv:hep-lat/0511007.

[16] P.O. Bowman, U.M. Heller, D.B. Leinweber, A.G. Williams, Nucl. Phys. B (Proc. Suppl.) 119 (2003) 323, arXiv:hep-lat/0209129; 
P.O. Bowman, U.M. Heller, A.G. Williams, Phys. Rev. D 66 (2002) 014505, arXiv:hep-lat/0203001.

[17] S. Furui, H. Nakajima, Phys. Rev. D 73 (2006) 074503.

[18] R.D. Bowler, M.C. Birse, Nucl. Phys. A 582 (1995) 655, arXiv:hep-ph/9407336;

R.S. Plant, M.C. Birse, Nucl. Phys. A 628 (1998) 607, arXiv:hep-ph/9705372.

[19] A. Scarpettini, D. Gómez Dumm, N.N. Scoccola, Phys. Rev. D 69 (2004) 114018, arXiv:hep-ph/0311030.

[20] D. Gómez Dumm, A.G. Grunfeld, N.N. Scoccola, Phys. Rev. D 74 (2006) 054026, arXiv:hep-ph/0607023.

[21] B. Golli, W. Broniowski, G. Ripka, Phys. Lett. B 437 (1998) 24, arXiv:hep-ph/9807261;

W. Broniowski, B. Golli, G. Ripka, Nucl. Phys. A 703 (2002) 667, arXiv:hep-ph/0107139.

[22] A.H. Rezaeian, N.R. Walet, M.C. Birse, Phys. Rev. C 70 (2004) 065203, arXiv:hep-ph/0408233;

A.H. Rezaeian, H.J. Pirner, Nucl. Phys. A 769 (2006) 35, arXiv:nucl-th/0510041.

[23] M. Praszalowicz, A. Rostworowski, Phys. Rev. D 64 (2001) 074003;

M. Praszalowicz, A. Rostworowski, Phys. Rev. D 66 (2002) 054002, arXiv:hep-ph/0111196

[24] S. Noguera, Int. J. Mod. Phys. E 16 (2007) 97, arXiv:hep-ph/0502171v5.

[25] S. Noguera, V. Vento, Eur. Phys. J. A 28 (2006) 227, arXiv:hep-ph/0505102.

[26] S. Noguera, N.N. Scoccola, Phys. Rev. D 78 (2008) 114002, arXiv:0806.0818 [hep-ph].

[27] See e.g. S. Noguera, V. Vento, Eur. Phys. J. A 46 (2010) 197, arXiv:1001.3075 [hep-ph].

[28] J. Prades, Z. Phys. C 63 (1994) 491, arXiv:hep-ph/9302246;

J. Prades, Z. Phys. C 11 (1999) 571 (Erratum).

[29] W. Broniowski, E.R. Arriola, Phys. Lett. B 649 (2007) 49, arXiv:hep-ph/0701243.

[30] P. Kotko, M. Praszalowicz, Phys. Rev. D 80 (2009) 074002, arXiv:0907.4044 [hep-ph]. 